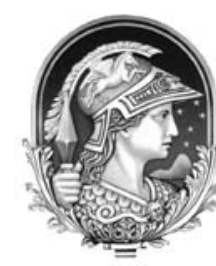

Anuário do Instituto de Geociências - UFRJ

\title{
Relação entre o Multivariate Enso Index (MEI) e a TSM das Regiões dos Niños com a Precipitação em Regiões Homogêneas do Estado do Rio Grande do Sul
}

Relationship Between Multivariate Enso Index (MEI) and SST of Niños Regions with the Precipitation in the Homogeneous Regions on Rio Grande do Sul State

\author{
Fábio Ziemann Lopes ${ }^{1}$; Gilberto Barbosa Diniz ${ }^{2} \&$ Júlio Renato Marques $^{3}$ \\ ${ }^{1}$ Universidade Federal de Pelotas - Curso de Pós graduação em Meteorologia \\ Av. Idelfonso Simões Lopes, 2751 , Pelotas, RG, 96020-290 \\ E-mail:fzlopes@bol.com.br \\ ${ }^{2}$ Universidade Federal de Pelotas - Curso de Pós graduação em Meteorologia \\ Av. Idelfonso Simóes Lopes, 2751, Pelotas, RG,96020-290 \\ E-mail: gilberto@ufpel.edu.br \\ ${ }^{3}$ Universidade Federal de Pelotas - Curso de Pós graduação em - Av. Idelfonso Simões Lopes, 2751, Pelotas, RG, $96020-290$ \\ E-mail: jmarques_fmet@ufpel.edu.br \\ Recebido em: 27/06/2007 Aprovado em: 10/08/2007
}

\section{Resumo}

No Rio Grande do Sul, as estações do ano são bem definidas sendo sentidos, em suas características peculiares, o inverno, a primavera, o verão e o outono. O regime pluviométrico é bastante regular e as chuvas são bem distribuídas durante todo o ano no Estado. O Índice Multivariado do El Niño Oscilação Sul (MEI) carece de um estudo de sua relação com a precipitação. Ele é um índice numérico que integra a ação de diferentes fatores que caracterizam o fenômeno e que oscila entre valores positivos para a fase quente, o El Niño, e valores negativos para a fase fria, a La Nina. Considera na sua composição, as seguintes variáveis: pressão ao nível do mar, as componentes zonal e meridional do vento em superfície, a Temperatura da Superfície do Mar (TSM), a temperatura do ar em superfície e um indicador de nebulosidade. Este trabalho teve como objetivo, estudar as relações entre o MEI e a TSM das regiões dos Niños com as precipitações no Estado do Rio Grande do Sul. Para isso, foram utilizados dados de totais mensais de precipitação de 40 estações meteorológicas do Rio Grande do Sul, dados bimestrais do MEI e de TSM das regiões dos Niños para o período de 1950 a 2002 . Os coeficientes de correlação entre precipitação do Rio Grande do Sul com o MEI e as regiões dos Niños apresentaram valores baixos devido ao fato de se utilizar apenas os meses do início e final do evento. O MEI, apesar de ser um índice mais complexo do ponto de vista metodológico, não melhora os coeficientes de correlação com a precipitação do Estado do Rio Grande do Sul, e sempre apresenta valores menores ou iguais aos obtidos ao utilizar a TSM das regiões dos Niños nos bimestres de out/nov e nov/dez. O MEI e as regiões dos Niños 3, 3.4 são as que apresentam os maiores coeficientes de correlação com a precipitação do Rio Grande do Sul para os bimestres out/nov e nov/dez.

Palavras-chaves: El Niño; MEI; precipitação

\begin{abstract}
On Rio Grande do Sul the seasons of the year are well defined being felt, in its peculiar characteristics, in the winter, in the spring, in the summer and in the autumn. The pluviometric regime is quite regular and the precipitations are well distributed during all the year on the State. The Multivariate Enso Index (MEI) lacks of a study about its relations with the precipitation. It is a numeric index that integrates the action of different factors that characterize the phenomenon and that oscilate between positive values for the warm phase, the El Niño, and negative values for the cold phase, the La Niña. It considers, in its composition, the following variables: sea level pressure, zonal and meridional wind components at the surface, the Sea Surface Temperature (SST), the air temperature at the surface and a cloudiness indicator. This work had the objective to study the relations between the MEI and the SST of the Niño regions with the precipitations on Rio Grande do Sul State. For this, it were utilized total monthly data of precipitation from 40 meteorological stations of Rio Grande do Sul, bimonthly data of MEI and SST of the Niño regions for the period 1950 to 2002. The correlation coefficients between the precipitation of the Rio Grande Do Sul with the MEI and the regions of the Niños showed low values due to the fact of if using only the months of the beginning and end of the event. The MEI, although to be a more complex index of the methodologic point of view, it does not improve the coefficients of correlation with the precipitation of the State of the Rio Grande do Sul, and it always presents lesser or equal values to obtained when using the TSM of the regions of the Niños in the out/nov and nov/dez coupled of months. The MEI and the Niños regions 3 and 3.4 present the highest correlation coefficient with the Rio Grande do Sul State precipitation for the bimonths oct/nov and nov/dec.
\end{abstract}

Keyswords: El Niño; MEI; precipitation 


\section{Relação entre o Multivariate Enso Index (MEI) e a TSM das Regiões dos Niños com a Precipitação em \\ Regiões Homogêneas do Estado do Rio Grande do Sul \\ Fábio Ziemann Lopes; Gilberto Barbosa Diniz \& Júlio Renato Marques}

\section{Introdução}

No Rio Grande do Sul, as estações do ano são bem definidas sendo sentidos, em suas características peculiares, o inverno, a primavera, o verão e o outono. O regime pluviométrico é bastante regular e as precipitações são bem distribuídas durante todo o ano no Estado.

Com referência à estiagem, fenômeno de secas prolongadas, esta se apresenta em algumas regiões climáticas do Estado, em determinados anos. Este fenômeno tem certa intensidade, a ponto de ameaçar a economia com prejuízos em setores como a agricultura, pecuária, indústria e outros que afetam a população.

Segundo Moreno (1961), citado por Puchalski (2000) o clima do Rio Grande do Sul é classificado, segundo Köeppen, na zona fundamental temperada ou "C", tipo úmido ou "Cf", com variedades "Cfa" e "Cfb" (subtropical e temperado respectivamente).

O El Niño é um fenômeno atmosféricooceânico caracterizado por um aquecimento anormal das águas superficiais no Oceano Pacífico Tropical e que pode afetar o clima regional e global, mudando os padrões de vento em nível mundial e, afetando, assim, os regimes de chuva em regiões tropicais e de latitudes médias.

Talvez a melhor maneira de se referir ao fenômeno El Niño seja pelo uso da terminologia mais técnica, que inclui as características oceânicasatmosféricas, associadas ao aquecimento anormal do Oceano Pacífico Tropical. O ENOS ou El Niño Oscilação Sul representa, de forma mais genérica, um fenômeno de interação atmosfera-oceano, associado às alterações dos padrões de Temperatura da Superfície do Mar (TSM) e dos ventos alísios na região do Pacífico equatorial entre a Costa Peruana e no Pacífico Oeste próximo à Austrália.

Além de índices baseados nos valores da TSM no Oceano Pacífico equatorial, o fenômeno ENOS pode ser também quantificado pelo Índice de Oscilação Sul (IOS). Esse índice representa a diferença entre a pressão ao nível do mar entre o Pacífico central (Taiti) e o Pacífico do oeste (Darwin
/Austrália). Está relacionado com as mudanças na circulação da atmosfera, conseqüência do resfriamento /aquecimento das águas superficiais na região. Valores negativos e positivos do IOS são indicadores da ocorrência do El Niño e La Niña respectivamente. No Brasil, em anos El Nino, as regiões Norte e Nordeste sofrem com acentuadas secas enquanto que no $\mathrm{Sul}$ a precipitação ultrapassa os valores médios normais. As anomalias de TSM na região de ocorrência do El Niño e La Niña, são monitoradas dividindo a região afetada em sub-regiões. Cada uma recebe uma identificação que, segundo o Climate Prediction Center (CPC), é a seguinte: Niño $1+2\left(0^{\circ}-10^{\circ} \mathrm{S}\right)$ e $\left(90^{\circ} \mathrm{W}-80^{\circ} \mathrm{W}\right)$, Niño $3\left(5^{\circ} \mathrm{N}-5^{\circ} \mathrm{S}\right)$ e $\left(150^{\circ} \mathrm{W}-90^{\circ} \mathrm{W}\right)$, Niño $4\left(5^{\circ} \mathrm{N}-5^{\circ} \mathrm{S}\right)$ e $\left(160^{\circ} \mathrm{W}-150^{\circ} \mathrm{W}\right)$ e uma nova região intermediária entre (3) e (4) chamada de Niño $3.4\left(5^{\circ} \mathrm{N}-5^{\circ} \mathrm{S}\right)$ e (170 $\left.{ }^{\circ} \mathrm{W}-120^{\circ} \mathrm{W}\right)$ (Cavalcanti, 1996).

Segundo Sampaio (2001), por ser uma região agrícola muito importante e por ter a precipitação como um dos principais fatores na produção agrícola, o sul do Brasil e o Estado do Rio Grande do Sul despertam a atenção dos pesquisadores no desenvolvimento de trabalhos voltados para a precipitação, bem como nos fatores que a influenciam.

Dentre esses fatores, o Índice Multivariado do El Niño Oscilação Sul (MEI) pode ser um deles e necessita um estudo de sua relação com a precipitação. Ele é um índice numérico que integra a ação de diferentes fatores que caracterizam o fenômeno e que oscila entre valores positivos para a fase quente, o El Niño, e negativo para a fase fria, a La Niña, e que considera, na sua composição, as seguintes variáveis: pressão ao nível do mar, as componentes zonal e meridional do vento em superfície, a temperatura da superfície do mar, a temperatura do ar em superfície e um indicador de nebulosidade (Lay \& Aiello, 2001).

Buscou-se neste trabalho:

Estudar as relações entre o MEI e a TSM das regiões dos Niños com as precipitações no Estado do Rio Grande do Sul.

Estudar as relações da TSM das regiões do Niños com as precipitações em estações meteorológicas do Rio Grande do Sul.

Verificar as relações entre o MEI e a TSM das regiões dos Niños com as precipitações em regiões homogêneas do Rio Grande do Sul. 


\section{Relação entre o Multivariate Enso Index (MEI) e a TSM das Regiões dos Niños com a Precipitação em \\ Regiões Homogêneas do Estado do Rio Grande do Sul \\ Fábio Ziemann Lopes; Gilberto Barbosa Diniz \& Júlio Renato Marques}

\section{2 Índice Multivariado do ENOS (MEI)}

Neste trabalho, pretende-se fundamentar o MEI para seis variáveis principais observadas no Pacífico Tropical, região de monitoramento do ENOS (El Niño-Oscilação Sul), fenômeno oceânicoatmosférico que influencia no clima global e nas escalas interanuais de tempo. Estas seis variáveis, computadas pelo COADS (Comprehensive Ocean - Atmosphere Data Set), NOAA (National Oceanic and Atmospheric Administration), são: pressão ao nível médio do mar, componente zonal e meridional do vento em superfície, Temperatura da Superfície do Mar, temperatura do ar em superfície e uma fração de nebulosidade. Para valores negativos do MEI, é observada a fase fria do ENOS (La Niña) e, para valores positivos, a fase quente (El Niño). O MEI foi calculado separadamente, para cada um dos doze meses do ano, e expresso como médias bimestrais (Dez-jan, Jan-fev,..., Nov-dez). Este índice é obtido usando a primeira Componente Principal $(\mathrm{CP})$ não rotacionada de todos os seis campos observados e combinados. Inicialmente, é normalizada a variação total de cada campo e, então, executada a extração do primeiro CP na matriz de covariância dos campos combinados (Lopes, 2006).

\subsection{Regiões dos Niños}

Devido à extensão muito grande da região do Pacífico Equatorial responsável pela anomalia da Temperatura da Superfície do Mar (TSM), esta é divida em partes cuja variação de temperatura tem influência diferente nas condições climáticas do Rio Grande do Sul. A conforme figura 1 mostra essas regiões.

\subsection{Relação entre o ENOS e a Precipitação}

A seguir, veremos alguns trabalhos que tratam do El Niño, La Niña, e sua relação com a precipitação, com a Temperatura da Superfície do Mar (TSM), e com o Multivariate Enso Index (MEI), e alguns, com aplicações na agricultura.

Baldo et al. (2000) analisaram através de dados da ANEEL (Agência Nacional de Energia Elétrica) para um período de 1950 a 1997 a variabilidade anual e interanual de precipitação para o Estado de Santa Catarina; o índice padronizado de precipitação foi correlacionado com a anomalia da TSM do Pacífico. Este cálculo foi efetuado para todo o Estado de Santa Catarina e concluíram que a Oscilação Sul está

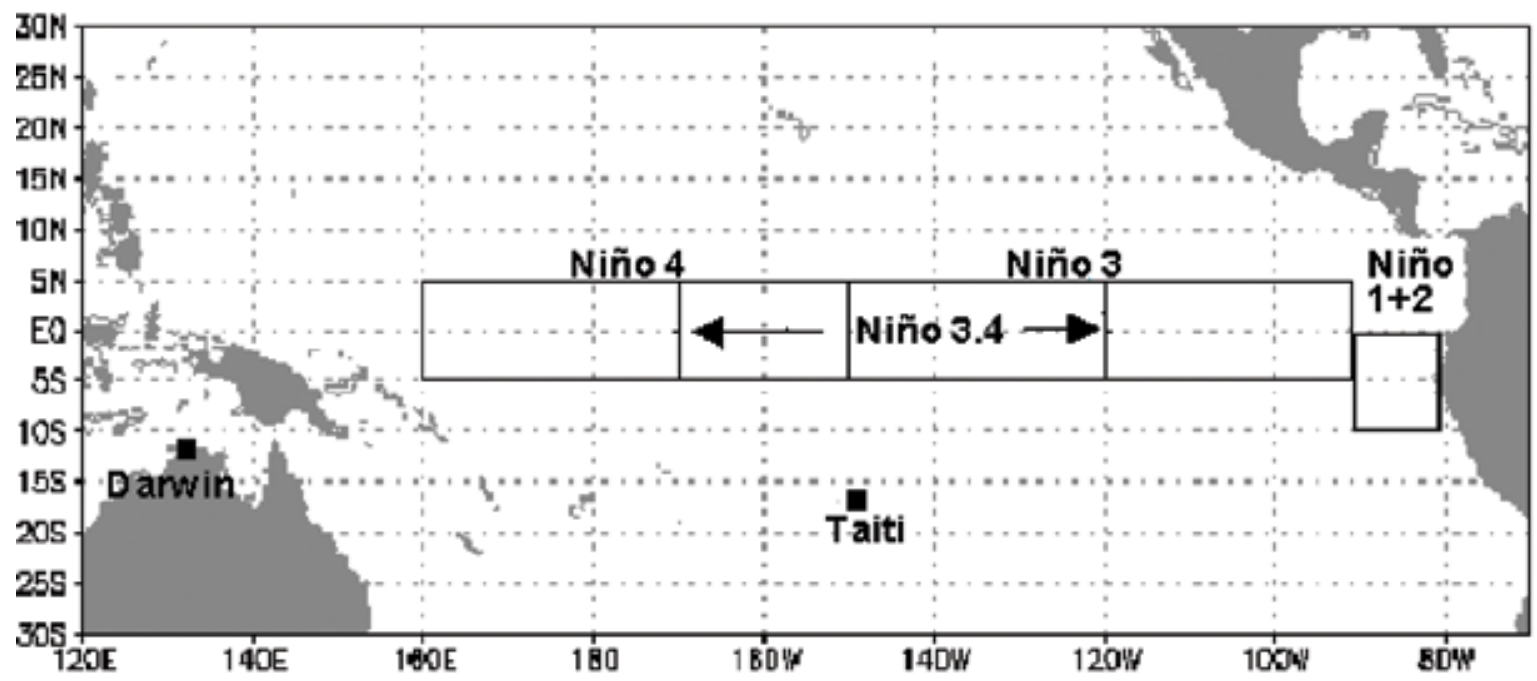

Figura 1 Regiões dos Niños no Pacífico equatorial. Fonte: <http://tucupi.cptec.inpe.br/enos/> Acessado em 27 de agosto de 2004. 


\section{Relação entre o Multivariate Enso Index (MEI) e a TSM das Regiões dos Niños com a Precipitação em \\ Regiões Homogêneas do Estado do Rio Grande do Sul \\ Fábio Ziemann Lopes; Gilberto Barbosa Diniz \& Júlio Renato Marques}

relacionada à anomalia da precipitação no Estado, especialmente durante anos El Niño.

Baptista \& Berlato (2003), avaliaram a influência do fenômeno ENOS no rendimento médio de grãos de feijão safra do Estado do Rio Grande do Sul (RS). Este trabalho foi realizado com dados de rendimentos de grãos de feijão safra do RS, no período de 1975/76 a 2000/01 (IBGE e FEE), no mesmo período, os dados de precipitação foram obtidos de estações meteorológicas pertencentes à FEPAGRO e ao $8^{\circ}$ DISME/INMET. Foram calculados os desvios dos rendimentos em relação à média do período $\mathrm{e}$ estes relacionados com o fenômeno ENOS; os anos de ocorrência de eventos ENOS foram considerados conforme Tremberth (1997) incluindo-se na lista os eventos de La Niña de 1998/99 e 1999/00. Através disso, constataram que os eventos La Niña foram favoráveis ao rendimento de feijão no Estado, já os eventos El Niño são os que apresentam maiores riscos de quebra de safra de feijão no Estado.

Berlato \& Fontana (1999) relacionaram a variabilidade interanual de precipitação com a variabilidade interanual do rendimento médio de grãos de soja do Estado do Rio Grande do Sul à luz do fenômeno El Niño Oscilação Sul. Os dados do rendimento médio de grãos de soja do Estado para o período 1974/75 a 1994/95 foram obtidos de estatísticas oficiais (Anuário Estatístico do Brasil, 1975-95) e os dados de precipitação mensal, desse mesmo período e do período 1913-94, obtidos de estações meteorológicas pertencentes ao $8^{\circ}$ Distrito de Meteorologia do Instituto Nacional de Meteorologia ( $8^{\circ}$ DISME/INMET).Com isso, concluíram que há fortes evidências que o fenômeno El Niño favorece a cultura da soja, determinado, em vários casos, rendimentos recordes dessa oleaginosa no Estado.

Coelho \& Ambrizzi (2000) analisaram através de técnicas estatísticas a influência da TSM da bacia do pacífico equatorial na precipitação sobre a América do Sul. A análise foi feita de modo que a divisão das regiões de influência aparecesse naturalmente, como resultado dos métodos empregados. Foram utilizados dados mensais de precipitação de uma série de estações meteorológicas e dados mensais da temperatura da superfície do mar (TSM). Tiveram como resultado que a região central do pacífico equatorial entre $100^{\circ} \mathrm{W}$ e $140^{\circ} \mathrm{W}$ dentro da região Niño 3 exerce maior influência sobre os padrões de precipitação da América do Sul durante o evento ENOS.
Utilizando dados mensais de precipitação de 206 estações meteorológicas de superfície da Rede Nacional do INMET (Instituto Nacional de Meteorologia), relativos ao período de 19601998 Coelho et al. (1999), realizaram um estudo climatológico sazonal das anomalias de precipitação acumulada sobre o Brasil, em casos caracterizados por eventos extremos de Oscilação Sul (OS). Com isso, concluíram que para um dado trimestre, há distinções nas características do padrão de precipitação entre os eventos forte/ moderado e fraco e para o evento El Niño 1997/1998, as observações mostram anomalias positivas ao sul e negativas ao norte do país.

Cunha et al. (2001) avaliaram o impacto das fases do fenômeno ENOS no rendimento de grãos da cultura de cevada no Brasil.Os dados foram registros históricos de rendimento da cevada do período de 1938 a 1998. Foram estudados quanto à sua variabilidade em relação as fases do fenômeno El Niño - Oscilação Sul (El Niño e La Niña e anos neutros). Os dados de rendimento médio anual de cevada $(\mathrm{kg} / \mathrm{ha})$ foram agregados por Estado (Rio Grande do Sul, Santa Catarina e Paraná). Eles chegaram à conclusão que o fenômeno El Niño - Oscilação Sul influencia o rendimento de grãos da cultura de cevada no país e na maioria das vezes, causa impactos positivos nos anos de La Niña e impactos negativos nos anos de El Niño, particularmente na região sul do país.

Diniz \& Puchalski (2000) estudaram a influência do El Niño Oscilação Sul na precipitação da região missioneira do Rio Grande do Sul, usando a técnica de dados denominada diagrama de caixa ("Box Plot") visando melhor entender a climatologia, variabilidade e previsibilidade desta variável. Como existiam falhas nas séries climatológicas de precipitação foi feita uma recomposição das mesmas utilizando o método proposto por Gonçalves \& Kim (1998) o qual utiliza a técnica multivariada de regressão linear múltipla no preenchimento das falhas existentes nas séries. Concluíram que a análise de distribuição da precipitação mostrou que o fenômeno El Niño tem uma marcante influência sobre a precipitação na região Missioneira, com exceção da região de Santiago que está localizada mais ao Sul da região.

Diniz et al. (1998) estudaram a influência do El Niño Oscilação Sul nas precipitações mensais de Pelotas para o período de 1950 a 1997, visando melhor entender a climatologia, variabilidade e mesmo previsibilidade potencial desta variável. Com isso 


\section{Relação entre o Multivariate Enso Index (MEI) e a TSM das Regiões dos Niños com a Precipitação em \\ Regiões Homogêneas do Estado do Rio Grande do Sul \\ Fábio Ziemann Lopes; Gilberto Barbosa Diniz \& Júlio Renato Marques}

verificaram que nem todos os eventos El Niño contribuíram para o aumento das precipitações na região tendo como fator predominante a época do ano.

Fontana \& Berlato (1997) quantificaram a influência do fenômeno ENOS sobre a precipitação mensal para o Estado Rio Grande do Sul. Para isso, utilizaram dados de precipitação mensal de 29 estações meteorológicas pertencentes ao $8^{\circ}$ Distrito de Meteorologia do Instituto Nacional de Meteorologia (INMET). O período de observação foi de 1913 e 1995. Com isso verificaram que existe influência do fenômeno ENOS sobre a distribuição anual da precipitação no Estado do Rio Grande do Sul e a influência maior é na porção oeste do Estado e nos meses de outubro e novembro meses em que um adequado suprimento hídrico é muito importante para o estabelecimento das culturas de primavera-verão.

Lay \& Aiello (2001) realizaram uma correlação simples (r) entre somas anuais de precipitação pontuais na Argentina Central entre 1950 e 1999 e médias anuais do Índice Multivariado do El niño Oscilação Sul (MEI) dados estes disponíveis desde 1950. Em alguns resultados $r$ superara o valor de 0,60 .

Sansigolo et al. (2004) estudaram as relações simultâneas e defasadas entre as categorias de precipitação em grupos homogêneos na região sul do Brasil e as categorias de TSM, nos oceanos Pacífico e Atlântico. Utilizaram para tanto tabelas de contingência $3 \times 3$ avaliadas pelos escores das características operacionais relativas. Para este estudo foram utilizados os totais mensais de precipitação referentes a 61 estações pluviométricas bem distribuídas na região sul do Brasil, no período de janeiro de 1950 a dezembro de 1997. Com isso concluíram que a maior influência dos eventos de TSM no Pacífico (El Niño e La Niña) nas respectivas categorias (acima e abaixo da normal) ocorreu no $4^{\circ}$ trimestre do ano e que a maior influência no Atlântico foi a definida como sudoeste e ocorreu predominantemente no $2^{\circ}$ trimestre do ano, nas categorias de TSM e precipitação acima da normal.

\section{Caracterização da Área de Estudo}

O Estado do Rio Grande do Sul localiza-se entre as latitudes $27^{\circ} 05^{\prime}$ e $33^{\circ} 45^{\prime}$ Sul e longitudes de $49^{\circ}$ $43^{\prime}$ e $57^{\circ} 39^{\prime}$ Oeste, tendo a área total de $282.184 \mathrm{Km}^{2}$, com uma costa marítima de $600 \mathrm{Km}$ de extensão. As altitudes no Estado variam do nível médio do mar junto à costa litorânea do Oceano Atlântico até $1.300 \mathrm{~m}$ em alguns pontos da Região do Planalto Superior (nordeste do Estado). Os principais mecanismos causadores da precipitação no Rio Grande do Sul são as passagens de sistemas frontais e o desenvolvimento de complexos convectivos de mesoescala (CCM's). A precipitação total anual do Estado é de $1.540 \mathrm{~mm}$, sendo que, no verão, outono, inverno e primavera ocorrem $24 \%, 25 \%, 25 \%$ e $26 \%$ deste total anual respectivamente (Puchalski, 2000).

\subsection{Dados Meteorológicos e Período de Observação}

Foram usados três conjuntos de dados de variáveis meteorológicas. O primeiro refere-se aos totais mensais de precipitação de 40 estações meteorológicas do Rio Grande do Sul para o período de 1950 a 2002. Estas estações estão mostradas na Tabela 1. Como poucas estações apresentavam o período completo de dados sem falhas, os dados faltantes foram preenchidos utilizando o método proposto por Gonçalves \& Kim (1998), o qual usa a técnica de regressão linear múltipla.

O segundo conjunto de informações foram dados bimestrais do MEI (Multivariate Enso Index) para o mesmo período, totalizando uma série de 53 anos.

E o terceiro são dados de Temperatura da Superfície do Mar (TSM) das regiões dos Niños para o mesmo período. Os dados das estações meteorológicas foram obtidos junto ao Instituto Nacional de Meteorologia - INMET ( $8^{\circ}$ Distrito de Meteorologia - Porto Alegre) do Ministério da Agricultura, Pecuária e Abastecimento (MAPA) e Fundação Estadual de Pesquisas Agropecuárias (FEPAGRO), da Secretaria de Ciência e Tecnologia (SCT) do Estado do Rio Grande do Sul. Já os de MEI e da Temperatura da Superfície do Mar (TSM) das regiões dos Niños, foram obtidos junto a NOAA (National Oceanic and Atmospheric Administration), através do site http://www.cdc.noaa.gov. 
Relação entre o Multivariate Enso Index (MEI) e a TSM das Regiões dos Niños com a Precipitação em

Regiões Homogêneas do Estado do Rio Grande do Sul

Fábio Ziemann Lopes; Gilberto Barbosa Diniz \& Júlio Renato Marques

\begin{tabular}{|c|c|c|c|c|}
\hline Estaçũo & Latitude & Longitude & Altitude (m) & Fonte \\
\hline 1-Alegrete & $29^{\circ} 41^{\prime}$ & $55^{\circ} 31^{\prime}$ & 124 & $8^{\circ}$ DISME \\
\hline 2-Bagé & $31^{\circ} 21^{\prime}$ & $54^{\circ} 06^{\prime}$ & 215 & $8^{\circ}$ DISME \\
\hline 3-Bento Gonçalves & $29^{\circ} 15^{\prime}$ & $51^{\circ} 31^{\prime}$ & 619 & $8^{\circ}$ DISME \\
\hline 4-Bom Jesus & $28^{\circ} 40^{\prime}$ & $50^{\circ} 26^{\prime}$ & 1047 & $8^{\circ}$ DISME \\
\hline 5-Caçapava do Sul & $30^{\circ} 30^{\prime}$ & $53^{\circ} 29^{\prime}$ & 450 & $8^{\circ}$ DISME \\
\hline 6-Cachoeira do Sul & $30^{\circ} 02^{\prime}$ & $52^{\circ} 53^{\prime}$ & 72 & $8^{\circ}$ DISME \\
\hline 7-Caxias do Sul & $29^{\circ} 10^{\prime}$ & $51^{\circ} 12^{\prime}$ & 785 & $8^{\circ}$ DISME \\
\hline 8-Cruz Alta & $28^{\circ} 38^{\prime}$ & $53^{\circ} 36^{\prime}$ & 472 & $8^{\circ}$ DISME \\
\hline 9-Encruzilhada do Sul & $30^{\circ} 32^{\prime}$ & $52^{\circ} 31^{\prime}$ & 427 & $8^{\circ}$ DISME \\
\hline 10-Farroupilha & $29^{\circ} 14^{\prime}$ & $51^{\circ} 26^{\prime}$ & 702 & FEPAGRO \\
\hline 11-Guaporé & $28^{\circ} 55^{\prime}$ & $51^{\circ} 54^{\prime}$ & 772 & $8^{\circ}$ DISME \\
\hline 12-Ijui & $28^{\circ} 23^{\prime}$ & $53^{\circ} 54^{\prime}$ & 448 & FEPAGRO \\
\hline 13-Irai & $27^{\circ} 11^{\prime}$ & $53^{\circ} 14^{\prime}$ & 222 & $8^{\circ}$ DISME \\
\hline 14-Júlio de Castilhos & $29^{\circ} 13^{\prime}$ & $53^{\circ} 40^{\prime}$ & 516 & FEPAGRO \\
\hline 15-Lagoa Vermelha & $28^{\circ} 25^{\prime}$ & $51^{\circ} 35^{\prime}$ & 836 & $8^{\circ}$ DISME \\
\hline 16-Marcelino Ramos & $27^{\circ} 27^{\prime}$ & $51^{\circ} 54^{\prime}$ & 414 & $8^{\circ}$ DISME \\
\hline 17-Osório & $29^{\circ} 41^{\prime}$ & $50^{\circ} 13^{\prime}$ & 32 & FEPAGRO \\
\hline 18-Palmeira das Missōes & $27^{\circ} 53^{\prime}$ & $53^{\circ} 26^{\prime}$ & 634 & $8^{\circ}$ DISME \\
\hline 19-Passo Fundo & $28^{\circ} 15^{\prime}$ & $52^{\circ} 24^{\prime}$ & 676 & $8^{\circ}$ DISME \\
\hline 20-Pelotas & $31^{\circ} 52^{\prime}$ & $52^{\circ} 21^{\prime}$ & 13 & $8^{\circ}$ DISME \\
\hline 21-Porto Alegre & $30^{\circ} 01^{\prime}$ & $51^{\circ} 13^{\prime}$ & 46 & $8^{\circ}$ DISME \\
\hline 22-Rio Grande & $32^{\circ} 01^{\prime}$ & $52^{\circ} 05^{\prime}$ & 5 & $8^{\circ}$ DISME \\
\hline 23-Santa Cruz do Sul & $29^{\circ} 43^{\prime}$ & $52^{\circ} 25^{\prime}$ & 56 & $8^{\circ}$ DISME \\
\hline 24-Santa Maria & $29^{\circ} 42^{\prime}$ & $53^{\circ} 42^{\prime}$ & 95 & $8^{\circ}$ DISME \\
\hline 25-Santa Rosa & $27^{\circ} 51^{\prime}$ & $54^{\circ} 25^{\prime}$ & 360 & $8^{\circ}$ DISME \\
\hline 26-Santa Vitória do Palmar & $33^{\circ} 31^{\prime}$ & $53^{\circ} 21^{\prime}$ & 6 & $8^{\circ}$ DISME \\
\hline 27-Santana do Livramento & $30^{\circ} 53^{\prime}$ & $55^{\circ} 32^{\prime}$ & 210 & $8^{\circ}$ DISME \\
\hline 28-Santiago & $29^{\circ} 11^{\prime}$ & $54^{\circ} 53^{\prime}$ & 426 & $8^{\circ}$ DISME \\
\hline 29-Santo Ângelo & $28^{\circ} 18^{\prime}$ & $54^{\circ} 15^{\prime}$ & 289 & $8^{\circ}$ DISME \\
\hline 30-São Borja & $28^{\circ} 39^{\prime}$ & $56^{\circ} 00^{\prime}$ & 96 & FEPAGRO \\
\hline 31-São Francisco de Paula & $29^{\circ} 20^{\prime \prime}$ & $51^{\circ} 30^{\prime}$ & 912 & $8^{\circ}$ DISME \\
\hline 32-São Gabriel & $30^{\circ} 20^{\prime}$ & $54^{\circ} 19^{\prime}$ & 124 & $8^{\circ}$ DISME \\
\hline 33-São Luiz Gonzaga & $28^{\circ} 23^{\prime}$ & $54^{\circ} 58^{\prime}$ & 254 & $8^{\circ}$ DISME \\
\hline 34-Soledade & $29^{\circ} 03^{\prime}$ & $52^{\circ} 26^{\prime}$ & 720 & $8^{\circ}$ DISME \\
\hline 35-Tapes & $30^{\circ} 52^{\prime}$ & $51^{\circ} 21^{\prime}$ & 5 & $8^{\circ}$ DISME \\
\hline 36-Taquari & $29^{\circ} 48^{\prime}$ & $51^{\circ} 49^{\circ}$ & 76 & $8^{\circ}$ DISME \\
\hline 37-Torres & $29^{\circ} 20^{\prime}$ & $49^{\circ} 43^{\prime}$ & 43 & $8^{\circ}$ DISME \\
\hline 38-Uruguaiana & $29^{\circ} 45^{\prime}$ & $57^{\circ} 05^{\prime}$ & 74 & $8^{\circ}$ DISME \\
\hline 39-Vacaria & $28^{\circ} 33^{\prime}$ & $50^{\circ} 42^{\prime}$ & 960 & $8^{\circ}$ DISME \\
\hline 40-Veranópolis & $28^{\circ} 56^{\prime}$ & $51^{\circ} 33^{\prime}$ & 705 & FEPAGRO \\
\hline
\end{tabular}

Tabela 1 Estações meteorológicas do Estado do Rio Grande do Sul, coordenadas geográficas e instituições a que pertence.

\subsection{Metodologia}

Como os dados do MEI obtidos do site da NOAA são bimestrais, foram feitas médias bimestrais dos totais mensais de precipitações das 40 estações meteorológicas do Rio Grande do Sul e da Temperatura da Superfície do Mar (TSM) nas regiões dos Niños. As séries de dados ficaram da seguinte forma: média bimestral de totais de precipitação e TSM das regiões dos Niños dos meses de jan-fev, fev-mar, mar-abr, abr-mai, mai-jun, jul-jul, jul-ago, ago-set, set-out, out-nov, nov-dez e dez-jan.
A fim de se verificar quais regiões pluviometricamente homogêneas do Estado apresentam maior coeficiente de correlação com MEI e a TSM das regiões dos Niños, foi feito um estudo semelhante para se obter essa relação entre a precipitação e estes índices. Para isso, foram utilizados dados do total médio bimestral da precipitação das estações que constituem seis regiões climaticamente homogêneas, ou seja, R1, R2, R3, R4, R5 e R6 obtidas do agrupamento seguindo o método e técnica proposta por Marques (2005). Esta técnica consiste num agrupamento levando em consideração a freqüência de precipitação como variável de agrupamento e o método utilizado foi o não hierárquico chamado k-média. 
Conhecendo-se as estações meteorológicas pertencentes as regiões descritas acima, calcularam-se as séries médias regionais bimestrais de precipitação para os bimestres jan-fev, fev-mar, mar-abr, abr-mai, mai-jun, jul-jul, jul-ago, ago-set, set-out, out-nov, novdez e dez-jan, as quais foram correlacionadas com as séries bimestrais do MEI e, também, com as séries médias bimensais da TSM das regiões dos Niños.

\section{Resultados e Discussão}

\subsection{Regiões Homogêneas do Rio Grande do Sul para a Precipitação.}

Seguindo o método de agrupamento utilizado por Marques (2005), conforme mapa da Figura 2, 6 regiões homogêneas foram obtidas. Estas seis regiões possuem juntas 40 estações meteorológicas. A região $\mathrm{R} 1$, é constituída por 4 estações meteorológicas, a R2 por 7, a R3 por 6, a R4 por 7, a R5 por 5 e a R6 por 11 estações. A Tabela 2 mostra as estações meteorológicas pertencentes a cada uma dessas regiões.

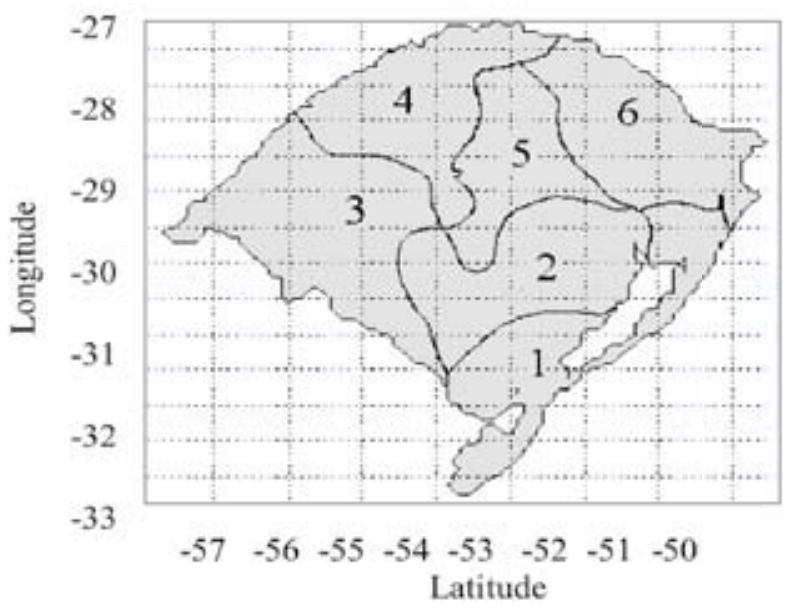

Figura 2 Regiões homogêneas de precipitação do Rio Grande do Sul.

\begin{tabular}{c|l|}
\hline Região & \multicolumn{1}{c|}{ Locais } \\
\hline R1 & $\begin{array}{l}\text { Osório, Pelotas, Rio Grande, e } \\
\text { Santa Vitória do Palmar. }\end{array}$ \\
\hline R2 & $\begin{array}{l}\text { Cachoeira do Sul, Encruzilhada do } \\
\text { Sul, Porto Alegre, Santa Cruz do } \\
\text { Sul, São Gabriel, Tapes e Taquari. }\end{array}$ \\
\hline R3 & $\begin{array}{l}\text { Alegrete, Bagé, Santana do } \\
\text { Livramento, Santiago São Borja e } \\
\text { Uruguaiana. }\end{array}$ \\
\hline R4 & $\begin{array}{l}\text { Cruz Alta, Ijui, Irai, Santa Maria, } \\
\text { Santa Rosa, Santo Ângelo, São } \\
\text { Luiz Gonzaga. }\end{array}$ \\
\hline R6 & $\begin{array}{l}\text { Caçapava do Sul, Júlio de } \\
\text { Castilhos, Palmeira das Missões, } \\
\text { Passo Fundo e Soledade. }\end{array}$ \\
$\begin{array}{l}\text { Bento Gonçalves, Bom Jesus, } \\
\text { Caxias do Sul, Farroupilha, } \\
\text { Guaporé, Lagoa Vermelha, } \\
\text { Marcelino Ramos, São Francisco } \\
\text { de Paula, Torres, Vacaria e } \\
\text { Veranópolis. }\end{array}$ \\
\hline
\end{tabular}

Tabela 2 Estações meteorológicas do Rio Grande do Sul, que fazem parte das regiões homogêneas de precipitação

\subsection{Relação entre a Precipitação das Regiões Homogêneas do Rio Grande do Sul e o MEI}

Para obter a relação entre o total médio bimestral da precipitação de cada região homogênea e o MEI foi calculado o coeficiente de correlação. Utilizando-se o Teste " $t$ " de Student, verificou-se que, para a dimensão das séries utilizadas, coeficientes de correlação $(r) \geq 0,25$ são significativos a $5 \%$, coeficientes de correlação $(r) \geq 0,30$ são significativos a $2 \%$ e coeficientes de correlação $(r) \geq 0,40$ são significativos a $1 \%$.

Aqui a mesma técnica foi aplicada para obter a relação entre o total médio bimestral da precipitação de cada região homogênea e o MEI. Essa relação foi calculada através do coeficiente de correlação entre elas. 


\begin{tabular}{|c|c|c|c|c|c|c|}
\hline Bimestres & R1 & R2 & R3 & R4 & R5 & R6 \\
\hline Jan-fev & 0,10 & 0,21 & $\mathbf{0 , 3 0}$ & $\mathbf{0 , 2 7}$ & 0,20 & 0,17 \\
Fev-mar & 0,13 & 0,23 & $\mathbf{0 , 3 1}$ & $\mathbf{0 , 3 1}$ & $\mathbf{0 , 3 1}$ & 0,12 \\
Mar-abr & 0,15 & 0,08 & 0,23 & 0,22 & 0,20 & 0,00 \\
Abr-mai & 0,22 & $\mathbf{0 , 2 7}$ & $\mathbf{0 , 3 7}$ & $\mathbf{0 , 4 2}$ & $\mathbf{0 , 3 2}$ & 0,24 \\
Mai-jun & 0,03 & $\mathbf{0 , 2 5}$ & $\mathbf{0 , 2 8}$ & $\mathbf{0 , 3 4}$ & 0,24 & 0,24 \\
Jun-jul & 0,17 & $\mathbf{0 , 2 7}$ & 0,15 & 0,19 & 0,15 & $\mathbf{0 , 2 5}$ \\
Jul-ago & 0,21 & $\mathbf{0 , 3 7}$ & 0,21 & $\mathbf{0 , 2 6}$ & $\mathbf{0 , 3 2}$ & $\mathbf{0 , 4 2}$ \\
Ago-set & 0,15 & 0,21 & 0,13 & 0,05 & 0,14 & 0,12 \\
Set-out & $\mathbf{0 , 3 6}$ & $\mathbf{0 , 3 4}$ & $\mathbf{0 , 2 9}$ & $\mathbf{0 , 2 8}$ & $\mathbf{0 , 2 8}$ & 0,21 \\
Out-nov & $\mathbf{0 , 6 0}$ & $\mathbf{0 , 5 9}$ & $\mathbf{0 , 6 0}$ & $\mathbf{0 , 6 0}$ & $\mathbf{0 , 6 3}$ & $\mathbf{0 , 6 2}$ \\
Nov-dez & $\mathbf{0 , 5 3}$ & $\mathbf{0 , 5 7}$ & $\mathbf{0 , 6 1}$ & $\mathbf{0 , 5 8}$ & $\mathbf{0 , 5 6}$ & $\mathbf{0 , 5 6}$ \\
Dez-jan & 0,15 & 0,15 & 0,23 & 0,09 & 0,00 & 0,10 \\
\hline
\end{tabular}

Tabela 3 Coeficientes de correlação (r) entre o MEI e os totais médios bimestrais da precipitação para regiões homogêneas do Estado do Rio Grande do Sul. Período 1950/2002

A Tabela 3 mostra o coeficiente de correlação (r) entre o MEI e os totais médios bimestrais da precipitação dos doze bimestres do ano para as 6 regiões homogêneas do Estado do Rio Grande do Sul.

Verificou-se coeficientes de correlação (r) significativos a pelo menos $5 \%$ em todas as regiões homogêneas em pelo menos 1 bimestre. Na região R1 nos bimestres: set-out, out-nov e nov-dez; na R2 nos bimestres: abr-mai, mai-jun, jun-jul, jul-ago, set-out, out-nov e nov-dez; na R3 jan-fev, fev-mar, abr-mai, mai-jun, set-out, out-nov e nov-dez; na R4 nos bimestres: jan-fev, fev-mar, abr-mai, mai-jun, julago, set-out, out-nov e nov-dez; na R5 nos bimestres: fev-mar, abr-mai, jul-ago, set-out, out-nov e nov-dez e na R6 nos bimestres: jun- jul, jul-ago, out-nov e novdez. Em todas as regiões homogêneas verificaram-se coeficientes de correlação (r) com valores maiores nos bimestres out-nov e nov-dez (bimestres estes que são referentes à primavera), sendo estes significativos a pelo menos $1 \%$.

\subsection{Relação entre a Precipitação das Regiões Homogêneas do Rio Grande do Sul e a TSM das Regiões dos Niños}

Logo após foi feita à relação entre o total médio bimestral da precipitação das regiões homogêneas do Rio Grande do Sul com a TSM das regiões dos Niños utilizando-se também o coeficiente de correlação (r), com as mesmas considerações do item 4.2.

\subsubsection{Niño $1+2$}

A Tabela 4 nos mostra o coeficiente de correlação (r) entre a média bimestral da TSM da região do Niño $1+2$ e os totais médios bimestrais da precipitação dos doze bimestres do ano para as 6 regiões homogêneas do Estado do Rio Grande do Sul.Verificou-se coeficientes de correlação (r) significativos a pelo menos $5 \%$ em todas as regiões homogêneas em pelo menos 1 bimestre. Na região R1 nos bimestres: out-nov e nov-dez; na R2 nos bimestres: out-nov e nov-dez; na R3 nos bimestres: jan-fev, abr-mai, out-nov, nov-dez e dez-jan; na R4 nos bimestres: jan-fev, fev-mar, abr-mai, out-nov e nov-dez; na R5 nos bimestres: jan-fev, fev-mar, abrmai, out-nov e nov-dez e na R6 nos bimestres: marabr, jul-ago, out-nov e nov-dez. Em todas as regiões homogêneas verificaram-se coeficientes de correlação (r) com valores maiores nos bimestres out-nov e novdez (bimestres estes que são referentes a primavera), sendo estes significativos a pelo menos $1 \%$.

\begin{tabular}{|c|c|c|c|c|c|c|}
\hline Bimestres & R1 & R2 & R3 & R4 & R5 & R6 \\
\hline Jan-fev & 0,12 & 0,22 & $\mathbf{0 , 3 5}$ & $\mathbf{0 , 3 8}$ & $\mathbf{0 , 3 3}$ & 0,24 \\
Fev-mar & $-0,05$ & 0,16 & 0,17 & $\mathbf{0 , 2 8}$ & $\mathbf{0 , 2 7}$ & 0,08 \\
Mar-abr & 0,16 & 0,18 & 0,24 & 0,23 & 0,21 & $\mathbf{0 , 2 7}$ \\
Abr-mai & 0,17 & 0,14 & $\mathbf{0 , 2 9}$ & $\mathbf{0 , 3 3}$ & $\mathbf{0 , 2 7}$ & 0,23 \\
Mai-jun & $-0,01$ & 0,12 & 0,19 & 0,22 & 0,09 & 0,01 \\
Jun-jul & 0,04 & 0,09 & 0,03 & 0,09 & 0,02 & 0,10 \\
Jul-ago & 0,15 & 0,21 & 0,06 & 0,18 & 0,23 & $\mathbf{0 , 4 0}$ \\
Ago-set & 0,23 & 0,12 & 0,03 & 0,03 & 0,14 & 0,14 \\
Set-out & 0,21 & 0,16 & 0,16 & 0,24 & 0,19 & 0,12 \\
Out-nov & $\mathbf{0 , 4 6}$ & $\mathbf{0 , 4 8}$ & $\mathbf{0 , 5 0}$ & $\mathbf{0 , 6 1}$ & $\mathbf{0 , 5 9}$ & $\mathbf{0 , 5 9}$ \\
Nov-dez & $\mathbf{0 , 5 0}$ & $\mathbf{0 , 5 2}$ & $\mathbf{0 , 5 5}$ & $\mathbf{0 , 5 3}$ & $\mathbf{0 , 4 9}$ & $\mathbf{0 , 4 7}$ \\
Dez-jan & 0,20 & 0,16 & $\mathbf{0 , 2 5}$ & 0,13 & $-0,03$ & 0,05 \\
\hline
\end{tabular}

Tabela 4 Coeficientes de correlação (r) entre a média bimestral da TSM da região do Niño $1+2$ e os totais médios bimestrais da precipitação do Estado do Rio Grande do Sul. Período 1950/2002

\subsubsection{Niño 3}

A Tabela 5 nos mostra o coeficiente de correlação (r) entre a média bimestral da TSM da região do Niño 3 e os totais médios bimestrais da precipitação dos doze bimestres do ano para as 6 regiões homogêneas do Estado do Rio Grande do Sul.

Verificou-se coeficientes de correlação (r) significativos a pelo menos $5 \%$ em todas as regiões 


\begin{tabular}{|c|c|c|c|c|c|c|} 
Bimestres & R1 & R2 & R3 & R4 & R5 & R6 \\
\hline Jan-fev & 0,16 & $\mathbf{0 , 2 5}$ & $\mathbf{0 , 3 7}$ & $\mathbf{0 , 3 5}$ & $\mathbf{0 , 3 1}$ & $\mathbf{0 , 2 5}$ \\
Fev-mar & 0,13 & 0,22 & $\mathbf{0 , 2 9}$ & $\mathbf{0 , 3 0}$ & $\mathbf{0 , 3 1}$ & 0,14 \\
Mar-abr & 0,14 & 0,11 & 0,20 & 0,19 & 0,18 & 0,11 \\
Abr-mai & 0,18 & 0,19 & $\mathbf{0 , 3 5}$ & $\mathbf{0 , 3 7}$ & $\mathbf{0 , 2 8}$ & 0,24 \\
Mai-jun & $-0,01$ & 0,12 & 0,21 & $\mathbf{0 , 3 0}$ & 0,18 & 0,11 \\
Jun-jul & 0,02 & 0,11 & 0,07 & 0,18 & 0,09 & 0,12 \\
Jul-ago & 0,06 & 0,23 & 0,15 & 0,20 & 0,24 & $\mathbf{0 , 3 0}$ \\
Ago-set & 0,21 & 0,23 & 0,21 & 0,10 & 0,20 & 0,13 \\
Set-out & $\mathbf{0 , 3 1}$ & $\mathbf{0 , 3 0}$ & $\mathbf{0 , 2 7}$ & $\mathbf{0 , 2 8}$ & $\mathbf{0 , 2 7}$ & 0,22 \\
Out-nov & $\mathbf{0 , 5 8}$ & $\mathbf{0 , 5 8}$ & $\mathbf{0 , 6 1}$ & $\mathbf{0 , 6 4}$ & $\mathbf{0 , 6 5}$ & $\mathbf{0 , 6 6}$ \\
Nov-dez & $\mathbf{0 , 5 5}$ & $\mathbf{0 , 5 7}$ & $\mathbf{0 , 6 4}$ & $\mathbf{0 , 6 2}$ & $\mathbf{0 , 5 6}$ & $\mathbf{0 , 5 3}$ \\
Dez-jan & 0,21 & 0,17 & $\mathbf{0 , 3 0}$ & 0,17 & 0,05 & 0,08 \\
\hline
\end{tabular}

Tabela 5 Coeficientes de correlação (r) entre a média bimestral da TSM da região do Niño 3 e os totais médios bimestrais da precipitação do Estado do Rio Grande do Sul. Período $1950 / 2002$

homogêneas em pelo menos 1 bimestre. Na região R1 nos bimestres: set-out, out-nov e nov-dez; na R2 nos bimestres: jan-fev, set-out, out-nov e nov-dez; na R3 nos bimestres jan-fev, fev-mar, abr-mai, set-out, outnov, nov-dez e dez-jan; na R4 nos bimestres: jan-fev, fev-mar, abr-mai, mai-jun, set-out, out-nov e nov-dez; na R5 nos bimestres: jan-fev, fev-mar, abr-mai, setout, out-nov e nov-dez e na R6 nos bimestres: janfev, jul-ago, out-nov e nov-dez. Em todas as regiões homogêneas verificaram-se coeficientes de correlação (r) com valores maiores nos bimestres out-nov e novdez (bimestres estes que são referentes a primavera), sendo estes significativos a pelo menos $1 \%$.

\subsubsection{Niño 3.4}

A Tabela 6 nos mostra o coeficiente de correlação (r) entre a média bimestral da TSM da região do Niño 3.4 e os totais médios bimestrais da precipitação dos doze bimestres do ano para as 6 regiões homogêneas do Estado do Rio Grande do Sul.

Verificou-se coeficientes de correlação (r) significativos a pelo menos $5 \%$ em todas as regiões homogêneas em pelo menos 1 bimestre. Na região R1 nos bimestres: set-out, out-nov e nov-dez; na R2 nos bimestres: jul-ago, ago-set, set-out, out-nov e nov-dez; na R3 nos bimestres: jan-fev, fev-mar, abr-mai, ago-set, set-out, out-nov e nov-dez; na R4 nos bimestres: jan-fev, fev-mar, abr-mai, mai-jun, set-out, out-nov e nov-dez; na R5 nos bimestres: fev-

\begin{tabular}{|c|c|c|c|c|c|c|}
\hline Bimestres & R1 & R2 & R3 & R4 & R5 & R6 \\
\hline Jan-fev & 0,11 & 0,21 & $\mathbf{0 , 2 9}$ & $\mathbf{0 , 2 7}$ & 0,24 & 0,19 \\
Fev-mar & 0,13 & 0,22 & $\mathbf{0 , 2 6}$ & $\mathbf{0 , 2 5}$ & $\mathbf{0 , 2 8}$ & 0,13 \\
Mar-abr & 0,15 & 0,02 & 0,15 & 0,07 & 0,08 & $-0,03$ \\
Abr-mai & 0,10 & 0,11 & $\mathbf{0 . 2 7}$ & $\mathbf{0 , 2 7}$ & 0,18 & 0,15 \\
Mai-jun & $-0,08$ & 0,11 & 019 & $\mathbf{0 , 2 7}$ & 0,21 & 0,18 \\
Jun-jul & 0,09 & 0,19 & 0,18 & 0,23 & 0,19 & 0,18 \\
Jul-ago & 0,03 & $\mathbf{0 , 2 6}$ & 0,24 & 0,20 & $\mathbf{0 , 2 5}$ & 0,24 \\
Ago-set & 0,20 & $\mathbf{0 , 2 6}$ & $\mathbf{0 , 2 7}$ & 0,10 & 0,20 & 0,11 \\
Set-out & $\mathbf{0 , 4 0}$ & $\mathbf{0 , 3 8}$ & $\mathbf{0 , 3 4}$ & $\mathbf{0 , 2 6}$ & $\mathbf{0 , 2 9}$ & 0,23 \\
Out-nov & $\mathbf{0 , 6 1}$ & $\mathbf{0 , 5 9}$ & $\mathbf{0 , 6 2}$ & $\mathbf{0 , 5 8}$ & $\mathbf{0 , 6 3}$ & $\mathbf{0 , 6 4}$ \\
Nov-dez & $\mathbf{0 , 5 3}$ & $\mathbf{0 , 5 5}$ & $\mathbf{0 , 6 3}$ & $\mathbf{0 , 5 8}$ & $\mathbf{0 , 5 4}$ & $\mathbf{0 , 5 2}$ \\
Dez-jan & $\mathbf{0 , 1 7}$ & $\mathbf{0 , 1 5}$ & $\mathbf{0 , 1 6}$ & 0,13 & 0,04 & 0,09 \\
\hline
\end{tabular}

Tabela 6 Coeficientes de correlação (r) entre a média bimestral da TSM da região do Niño 3.4 e os totais médios bimestrais da precipitação do Estado do Rio Grande do Sul. Período $1950 / 2002$

mar, jul-ago, set-out, out-nov e nov-dez e na R6 nos bimestres: out-nov e nov-dez. Em todas as regiões homogêneas verificaram-se coeficientes de correlação (r) com valores maiores nos bimestres out-nov e novdez (bimestres estes que são referentes a primavera), sendo estes significativos a pelo menos $1 \%$.

\subsubsection{Niño 4}

A Tabela 7 nos mostra o coeficiente de correlação (r) entre a média bimestral da TSM da região do Niño 4 e os totais médios bimestrais da precipitação dos doze bimestres do ano para as 6 regiões homogêneas do Estado do Rio Grande do Sul.

\begin{tabular}{|c|c|c|c|c|c|c|}
\hline Bimestres & R1 & R2 & R3 & R4 & R5 & R6 \\
\hline Jan-fev & 0,00 & 0,13 & 0,15 & 0,12 & 0,10 & 0,11 \\
Fev-mar & 0,09 & 0,17 & 0,19 & 0,11 & 0,14 & 0,02 \\
Mar-abr & 0,16 & $-0,02$ & 0,11 & $-0,04$ & $-0,02$ & $-0,16$ \\
Abr-mai & 0,01 & 0,00 & 0,15 & 0,07 & 0,01 & $-0,03$ \\
Mai-jun & $-0,11$ & 0,09 & 0,14 & 0,20 & 0,18 & 0,20 \\
Jun-jul & 0,17 & 0,31 & $\mathbf{0 , 2 6}$ & $\mathbf{0 , 2 7}$ & $\mathbf{0 , 2 7}$ & $\mathbf{0 , 2 9}$ \\
Jul-ago & 0,08 & 0,29 & 0,22 & 0,18 & 0,23 & 0,23 \\
Ago-set & 0,06 & 0,15 & 0,14 & 0,01 & 0,09 & 0,02 \\
Set-out & $\mathbf{0 , 4 1}$ & $\mathbf{0 , 4 1}$ & $\mathbf{0 , 3 6}$ & 0,20 & $\mathbf{0 , 2 7}$ & 0,21 \\
Out-nov & $\mathbf{0 , 6 2}$ & $\mathbf{0 , 6 0}$ & $\mathbf{0 , 6 2}$ & $\mathbf{0 , 4 9}$ & $\mathbf{0 , 5 8}$ & $\mathbf{0 , 5 6}$ \\
Nov-dez & $\mathbf{0 , 5 1}$ & $\mathbf{0 , 5 3}$ & $\mathbf{0 , 5 5}$ & $\mathbf{0 , 4 5}$ & $\mathbf{0 , 4 7}$ & $\mathbf{0 , 4 7}$ \\
Dez-jan & 0,14 & $\mathbf{0 , 1 1}$ & 0,17 & 0,02 & $-0,02$ & $-0,03$ \\
\hline
\end{tabular}

Tabela 7 Coeficientes de correlação (r) entre a média bimestral da TSM da região do Niño 4 e os totais médios bimestrais da precipitação do Estado do Rio Grande do Sul. Período $1950 / 2002$ 


\section{Relação entre o Multivariate Enso Index (MEI) e a TSM das Regiões dos Niños com a Precipitação em Regiões Homogêneas do Estado do Rio Grande do Sul \\ Fábio Ziemann Lopes; Gilberto Barbosa Diniz \& Júlio Renato Marques}

Verificou-se coeficientes de correlação (r) significativos a pelo menos $5 \%$ em todas as regiões homogêneas em pelo menos 1 bimestre. Na região R1 nos bimestres: set-out, out-nov e nov-dez; na R2 nos bimestres: jun-jul, jul-ago, set-out, out-nov e nov-dez; na R3 nos bimestres: jun-jul, set-out, out-nov e novdez; na R4 nos bimestres: jun-jul, out-nov e nov-dez; na R5 nos bimestres: jun-jul, set-out, out-nov e novdez e na R6 nos bimestres: jun-jul, out-nov e novdez. Em todas as regiões homogêneas verificaram-se coeficientes de correlação (r) com valores maiores nos bimestres out-nov e nov-dez (bimestres estes que são referentes a primavera),sendo estes significativos a pelo menos $1 \%$.

A correlação entre a precipitação do Rio Grande do Sul e os índices (MEI e TSM das regiões dos Niños) apresentaram coeficiente de correlação não muito significativos em todas as regiões homogêneas no bimestre dez/jan.

\subsection{Análise da anomalia da precipitação nas regiões homogêneas para os bimestres out-nov e nov-dez}

Foram resumidos nas Tabelas 8,9 , os coefiecientes de correlação indicados nas tabelas dos itens anteriores para os bimestres out-nov e novdez. Estes dois bimestres foram escolhidos devido em todas as regiões homogêneas eles terem tido um grau de significância a pelo menos $1 \%$.

\begin{tabular}{|c|c|c|c|c|c|c|c|}
\hline & R1 & R2 & R3 & R4 & R5 & R6 & Média \\
\hline MEI & 0,60 & 0,59 & 0,60 & 0,60 & 0,63 & 0,62 & 0,606 \\
Niño 1+2 & 0,46 & 0,48 & 0,50 & 0,61 & 0,59 & 0,59 & 0,538 \\
Niño 3 & 0,58 & 0,58 & 0,61 & 0,64 & 0,65 & 0,66 & 0,620 \\
Niño 3.4 & 0,61 & 0,59 & 0,62 & 0,58 & 0,63 & 0,64 & 0,611 \\
Niño 4 & 0,62 & 0,60 & 0,62 & 0,49 & 0,58 & 0,56 & 0,593 \\
Média & 0,574 & 0,568 & 0,590 & 0,584 & 0,614 & 0,634 & \\
\hline
\end{tabular}

Tabela 8 Coeficientes de correlação entre índices e a precipitação nas regiões homogêneas para o bimestre out-nov

\begin{tabular}{|c|c|c|c|c|c|c|c}
\hline & R1 & R2 & R3 & R4 & R5 & R6 & Média \\
\hline MEI & 0,53 & 0,57 & 0,61 & 0,58 & 0,56 & 0,56 & 0,568 \\
Niño 1+2 & 0,50 & 0,52 & 0,55 & 0,53 & 0,49 & 0,47 & 0,51 \\
Niño 3 & 0,55 & 0,57 & 0,64 & 0,62 & 0,56 & 0,53 & 0,578 \\
Niño 3.4 & 0,53 & 0,55 & 0,63 & 0,58 & 0,54 & 0,52 & 0,558 \\
Niño 4 & 0,51 & 0,53 & 0,55 & 0,45 & 0,47 & 0,47 & 0,496 \\
Média & 0,524 & 0,54 & 0,59 & 0,55 & 0,52 & 0,51 & \\
& & 8 & 6 & 2 & 4 & 0 & \\
\hline
\end{tabular}

Tabela 9 Coeficientes de correlação entre índices e a precipitação nas regiões homogêneas para o bimestre nov-dez
Em estudos climáticos, utilizando o MEI e a TSM das regiões dos Niños relacionadas com a precipitação para o Estado do Rio Grande do Sul , recomenda-se utilizar neste prognóstico no bimestre out-nov: para a região R1, a TSM do Niño 4; para a região R2 a TSM do Niño 4; para a região R3 a TSM do Nino 3.4 ou a do Niño 4; para a região R4 a TSM do Niño 4; para a região R5 a TSM do Nino 3 e para a região $\mathrm{R} 6$ a TSM do 3.

Já para o bimestre nov-dez, recomenda-se utilizar neste prognóstico: Para a região R1, a TSM do Niño 3; para a região R2 o MEI ou a TSM do Niño 3; para a região R3 a TSM do Niño 3; para a região R4 a TSM do Niño 3; para a região R5 o MEI ou a TSM do Niño 3 e para a região R6 o MEI.

Já para os coeficientes de correlação entre os índices e a precipitação no Estado do Rio Grande do Sul no bimestre nov/dez o Niño 3 foi o que apresentou melhores resultados, concordando com Coelho \& Ambrizzi (2000).

O MEI não melhorou muito os resultados se comparado com a TSM das regiões dos Niños, por que foi calculado a partir da primeira componente principal não rotacionada.

Estes resultados confirmam, ampliam e quantificam os obtidos por Coelho \& Ambrizzi (2000) e Marques (2005).

\section{Conclusões}

A partir das análises feitas neste trabalho, de conformidade com os objetivos propostos, chegouse às seguintes conclusões: usando o coeficiente de correlação como uma medida de inter-relação entre as séries de dados que expressam o comportamento climatológico de variáveis meteorológicas pode-se concluir que:

a) O MEI, apesar de ser um índice mais complexo do ponto de vista metodológico, não melhora os coeficientes de correlação com a precipitação do Estado do Rio Grande do Sul, e sempre apresenta valores menores ou iguais aos obtidos ao utilizar a TSM das regiões dos Niños nos bimestres de out/nov e nov/dez. 


\section{Relação entre o Multivariate Enso Index (MEI) e a TSM das Regiões dos Niños com a Precipitação em \\ Regiões Homogêneas do Estado do Rio Grande do Sul \\ Fábio Ziemann Lopes; Gilberto Barbosa Diniz \& Júlio Renato Marques}

b) O MEI e as regiões dos Niños 3, 3.4 são as que apresentam os maiores coeficientes de correlação com a precipitação do Rio Grande do Sul para os bimestres out/nov e nov/dez.

\section{Referências}

Baldo, M. C.; Andrade, A. R.; Martins, ML. O. F. M. \& Nery, J. T. 2000. Análise da precipitação pluvial do Estado de Santa Catarina associada com a anomalia da temperatura da superfície do mar no oceano Pacífico. Revista Brasileira de Agrometeorologia, Santa Maria -RS, 8 (2): 283-293.

Baptista, R. L.\& Berlato, M. A. 2003. Impacto do El Niño e La Niña no rendimento de feijão safra do Rio Grande do Sul. In: CONGRESSO BRASILEIRO DE AGROMETEOROLOGIA, 13, Santa Maria. Anais... Santa Maria: SBA, 2003, p. 597-598.

Berlato, M. A. \& Fontana, D. C. 1999. Variabilidade interanual da precipitação pluvial e rendimento da soja no Estado do Rio Grande do Sul. Revista Brasileira de Agrometeorologia, Santa Maria -RS, 7 (1): 119-125.

Cavalcanti, I. F. A. 1996. Episódios El Niño/ Oscilação Sul durante a década de 1986 a 1996 e suas influências sobre o Brasil. In: Climanálise Especial: edição comemorativa de 10 anos. São José dos Campos-São Paulo, INPE/CPTEC, p.52-64.

Centro de Previsão do Tempo e Estudos Climáticos. 2004. Regiões do Niños no Pacífico Equatorial. Disponível em <http://tucupi.cptec.inpe.br/ enos/>. Acessado em 27 de ago. de 2004.

Coelho, C. A. S. \& Ambrizzi, T. 2000. Determinação dos padrões de precipitação sobre a América do Sul em associação a TSM da Bacia do Pacífico Equatorial durante períodos extremos... In: CONGRESSO BRASILEIRO DE METEOROLOGIA, 11, 2000, Rio de Janeiro. SBmet, 2000. CL-00003 1 CDROM.

Coelho, C. A. S.; Drumond, A. R. M.; Ambrizzi, T. \& Sampaio, G. 1999. Estudo climatológico sazonal da precipitação sobre o Brasil em episódios extremos de Oscilação Sul. Revista Brasileira de Meteorologia, Rio de Janeiro, 14 (1): 49-65.

Cunha, G.R.; Dalmago, G.A.; Estefanel, V.; Pasinato, A. \& Moreira, M. B. 2001. El Niño - Oscilação Sul e seus impactos sobre a cultura de cevada no
Brasil. Revista Brasileira de Agrometeorologia, Santa Maria -RS, 9 (1): 137-145.

Diniz, G. B.\& Puchalski , L. 2000. A. Utilização de diagrama de caixa (BOX PLOT) no estudo da influência do El-Niño na precipitação da Região Missioneira do Rio Grande do Sul. In: REUNION DE AGROMETEOROLOGIA, 8; Mendoza - Argentina, p. 88.

Diniz, G. B.; Saldanha, R. L.\& Sansigolo, C. A. 1998. Influência do evento El Niño no regime de precipitação de Pelotas, RS. In: CONGRESSO BRASILEIRO DE METEOROLOGIA, 10.; CONGRESSO DA FLISMET, 8., 1998, Brasília. Anais... Rio de Janeiro: SBMet, 1998. CL-98160. 1 CD-ROM.

Fontana, D. C \& Berlato, M. A. 1997. Influência do El Niño Oscilação Sul sobre a precipitação pluvial no Estado do Rio Grande do Sul. Revista Brasileira de Agrometeorologia, Santa Maria -RS , 5 (1): 127-132.

Gonçalves, F. V.\& Kim, I. S. 1998. Recuperação de dados meteorológicos do Rio Grande do Sul e Santa Catarina. In: CONGRESSO BRASILEIRO DE METEOROLOGIA, 10; CONGRESSO DA FLISMET, 1998, Brasília. Anais... Rio de Janeiro: SBmet, 1998. 1 CDROM.

Lay, J. A.\& Aiello, J. L. 2001. Relaciones entre el MEI (Multivariate ENSO Index) y las precipitaciones en la Argentina continental. In: CONGRESSO LATINO AMERICANO DE METEOROLOGIA, 9. CONGRESSO ARGENTINO DE METEOROLOGIA, 8. CLIMET 9, COMET 8, Buenos Aires del 7 al 11 de Mayo, 2001 CD-ROM.

Lopes, F. Z. 2006. Relação entre o MEI (Mulivariate ENSO Index) e a precipitação pluvial no Estado do Rio Grande do Sul. 2006. Dissertação de Mestrado-Meteorologia - Programa de Pósgraduação em Meteorologia, Faculdade de Meteorologia, Universidade Federal de Pelotas, Pelotas, $122 \mathrm{p}$.

Marques, J. R. 2005. Variabilidade espacial etemporal de precipitação pluvial no Rio Grande do Sul e sua relação com indicadores oceânicos. 2005. Tese de Doutorado-Agrometeorologia - Programa de Pós-graduação em Fitotecnia, Faculdade de Agronomia, Universidade Federal do Rio Grande do Sul, Porto Alegre, 210 p.

Moreno, J. A. 1961. Clima do Rio Grande do Sul. Porto Alegre: Secretária da Agricultura do Estado do Rio Grande do Sul, 30 p. 


\section{Relação entre o Multivariate Enso Index (MEI) e a TSM das Regiões dos Niños com a Precipitação em \\ Regiões Homogêneas do Estado do Rio Grande do Sul \\ Fábio Ziemann Lopes; Gilberto Barbosa Diniz \& Júlio Renato Marques}

National Oceanic and Atmospheric Administration 2004. Dados do MEI e da TSM das regiões dos Niños Disponível em: <http://www.cdc.noaa. gov $>$. Acessado em 30 de setembro de 2004.

Puchalski, L. A. 2000. Efeitos associados ao fenômeno El Niño e La Niña na temperatura média, precipitação pluvial e déficit hídrico no Estado do Rio Grande do Sul: Porto Alegre: UFRGS. Dissertação de Mestrado em Fitotecnia Agrometeorologia - Curso de Pós-graduação em Fitotecnia, Universidade Federal do Rio Grande do Sul, Porto Alegre, 99 p.
Sampaio, G. O. 2001. O El Niño e você-O fenômeno climático. São José dos Campos: Editora Transtec, $116 \mathrm{p}$.

Sansigolo, C. A.; Pereira, C. S. \& Silva, I. R. 2004. Relações entre as precipitações regionais no Sul do Brasil e as temperaturas das superfícies dos Oceanos Atlântico e Pacífico. Revista Brasileira de Meteorologia, Rio de Janeiro, 19 (1): 5-11.

Trenberth, K. E. 1997. The definition of El Niño. Bulletin of the American Meteorology Society, Washington. 78: 2771-2777. 